\title{
A MINI NUTRITIONAL ASSESSMENT OF THE RISK OF MALNUTRITION IN ELDERLY UNDER THREE NUTRITION SUPPORT MODELS
}

\author{
E. Lindhorst, M. Ramel, P. Kelly, L. Jones
}

\begin{abstract}
Objective: The aim of this study was to determine which nutritional support setting fostered the best nutritional status in elderly patients using the Mini Nutritional Assessment survey. Design and Participants: The analytical sample included a total of 75 adults aged 60-89 years. Setting: There were three nutrition support settings: a nursing home, an assisted living facility, and independent living with congregate feeding. Measurements: The Mini Nutritional Assessment was used to examine nutritional status in patients living in one of the three nutritional support settings. Results: Individuals living independently individuals and attending congregate feedings resulted in the most people in the "normal nutritional status" category when compared with nursing home and assisted living residents. Conclusion: Individuals living independently in their homes who use congregate feeding have reduced risk of malnutrition.
\end{abstract}

Key words: Mini Nutritional Assessment, nursing home, assisted living, independent living, congregate feeding.

\section{Introduction}

According to the U.S. Department of Health and Human Services, the elderly population is expected to increase about $19 \%$ by the year 2030 . Compared to $12.4 \%$ increase in the elderly population in 2000 , the United States is on a steady climb to having one of the largest elderly populations it has ever encountered (1). As individuals age, cognitive function gradually declines. This cognitive impairment may result in decreased nutrient intake, leading to poor nutritional status and possibly malnutrition. Cognitive decline, commonly known as dementia, can also result in a decrease in daily activities as well as behavioral and physiological changes. (2) It is estimated that by the year 2040, 81 million people will be affected by dementia (2).

As documented in previous studies, weight loss has been shown to increase the rate of functional decline and increase the risk of morbidity and mortality in the elderly (3). The presence of weight loss and physical decline is heavily correlated with increasing age (3). Therefore, malnutrition is prevalent in about $5-15 \%$ of the general elderly population. Studies suggest that institutionalized elderly have even greater rates of

Department of Nutrition and Dietetics, Saint Louis University, Saint Louis, Missouri.

Corresponding Author: Erin Lindhorst, Department of Nutrition and Dietetics, Saint Louis University, Allied Health Professions Building 3437 Caroline Street St. Louis, MO 63104, Tel. 816-210-2394, erinlindhorst@gmail.com malnutrition, where $52-82 \%$ of the population may suffer from malnourishment (4). Additionally, malnutrition often goes undetected in elderly living independently at home, with the prevalence of malnutrition ranging from $13-30 \%$ (5). Malnutrition is also seen more in adults with co-morbidities and on multiple prescription medications (4).

Increased mortality rates are correlated with malnutrition but can be prevented by nutritional screening and simple nutritional interventions (4). In order to assess malnutrition in elderly patients a multidimensional approach is needed (4). The Mini Nutritional Assessment (MNA) survey includes factors such as anthropometric measures, weight changes, dietary problems, motility issues, and neuropsychological status (4). The MNA survey consists of six questions that cover decline in food intake, weight loss during the last 3 months, mobility, psychological stress or acute disease, neuropsychological problems, and body mass index (BMI). The results of the assessment categorized individuals as "at normal nutritional status," "at risk of malnutrition," or "malnourished." Early detection of elderly individuals at nutritional risk, followed by nutritional intervention, can help to conserve muscle function as well as muscle strength, improve quality of life, and potentially prolong length of survival (6). Using the MNA survey can provide that early detection.

Nutrition status can vary between elderly patients 
living in different settings. There are three main settings where elderly tend to reside in as they age. These include: within a nursing home, in an assisted living facility, or within their home. Each of these living settings provides different nutrition support models. Within a nursing home, patients are provided care 24 hours a day. Skilled nurses or nursing aides can assist these patients with feeding, bathing and dressing if needed. These patients typically gather in a central dining area with other residents for meal times, but they are able to eat in their room if they so desire (7). An assisted living facility is for individuals who require minimal assistance and/or care with every day activities such as bathing, dressing and feeding. These individuals have access to a central dining area where they are provided meals, but they also have the option to prepare meals in their own kitchen (8). Elderly individuals who live independently but attend a congregate feeding site are usually able to perform activities of daily living without any assistance. They generally cook for themselves and potentially for other loved ones. Congregate feeding sites provide elderly individuals 60 years or above access to a meal 5 days a week. Along with a meal, these individuals also get social interaction and support from others attending the congregate feeding site. A review of current literature indicated that there was limited research on malnutrition prevalence in elderly living in these 3 nutrition support settings.

\section{Methods}

\section{Design}

The principal investigator (PI) found voluntary participants in a nursing home and recruited them during a gathering time where most residents were present. Persons in assisted living were recruited while they were gathered in the communal dining area of the residency. The participants living independently were recruited while they were at their congregate feeding site. Voluntary participants from each site were given a description of the research being conducted. The PI assessed them using the Mini Nutritional Assessment survey and 2 additional nutrition support questions. Surveys and additional questions were anonymous and all information collected throughout this study was kept confidential. The Saint Louis University Institutional Review Board approved the study.

\section{Sample}

Study participants were recruited from each of the following nutrition support settings: nursing home care, assisted living, or congregate feeding. Participants ranged in age from 60-89 years and had good cognitive status. The goal was to recruit 25 seniors from each nutrition support setting, for a total of 75 participants.

\section{Instrument}

The short form of the Mini Nutritional Assessment (MNA) and 2 additional questions were used to gather information during this research study. The MNA is a validated survey and detects whether or not an individual is malnourished or at risk of being malnourished. The survey consists of six questions that look at decline in food intake, weight loss during the past 3 months, mobility, psychological stress or acute disease, neuropsychological problems, and body mass index. The 2 additional questions that were asked depended on the nutrition support setting. The questions for the nursing home and assisted living participants were how long have you been living at this facility? Do you eat in your room or in the communal dining setting? The questions for the congregate feeding participants were how long have you been receiving nutrition support from the congregate feeding site? How many times a week do you receive meals at the facility? These questions were asked to determine if there was an association between nutritional status and length of residency, place where meals were eaten, and frequency of meals received under a nutrition support setting.

Anthropometrics were also required to complete this assessment. The most recent weight and height for the assisted living and nursing home residents were obtained from the facilities medical records. For congregate feeding participants, calf circumference (CC) was obtained using a standard measuring tape. According to the MNA survey, CC can be used in place of BMI if weight and height are unavailable.

\section{Data Analysis}

For the statistical analysis, Statistical Package for the Social Sciences (SPSS, 22.0, 2013) was used. The data were compared using descriptive statistics and analyzed using Chi-square tests of association. These tests were used to determine if there was an association between a participant's living facility and nutritional status.

\section{Results}

\section{Demographic Characteristics}

Table 1

Demographic Characteristics of Population Study

\begin{tabular}{lll}
\hline Characteristic & Number & Percent \\
\hline Age & & \\
$60-69$ & 20 & 26.7 \\
$70-79$ & 19 & 25.3 \\
$80-89$ & 48 & 48.0 \\
Gender & & \\
Female & 56 & 74.7 \\
& &
\end{tabular}




\begin{tabular}{lll} 
Male & 19 & 25.3 \\
Living Facility & & \\
Nursing home & 25 & 33.3 \\
Assisted living & 25 & 33.3 \\
Congregate feeding & 25 & 33.3 \\
Nutrition Score & & \\
Normal & 44 & 58.7 \\
At risk & 25 & 33.3 \\
Malnourished & 6 & 8.0 \\
\hline
\end{tabular}

\section{Comparison of Results between the Nutrition} Support Settings

This study compared the nutritional status of individuals living in a nursing home, an assisted living facility, and living independently at home but attending a congregate feeding site. The data analysis determined that individuals living independently but attending a congregate feeding site had the best nutritional status of the 3 groups. The individuals living independently while attending a congregate feeding site had the most participants in the "normal nutritional status" category. There were 20 individuals with "normal nutritional status" and 5 individuals "at risk or malnourished" (Table 2). The nursing home and assisted living participants had the same results. There were 12 individuals in the "normal nutritional status" category and 13 individuals in the "at risk or malnourished" category (Table 2). Using a Chi-square test to compare living facility and nutritional status showed a significant association between individual's living facility and their nutritional status (chi-square $=7.038(2), p<0.05)$ (Table 2).

\section{Discussion}

The purpose of this study was to compare nutritional status between elderly in 3 different nutritional support settings: a nursing home, an assisted living facility, and congregate feeding site. This study was conducted to determine which environment supported the best nutritional status as well as overall health status.

This study showed the independent living setting with access to congregate feeding resulted in lower risk of malnutrition in the elderly than the other two settings. The results of this study line up with previous studies that determined malnutrition rates increase in elderly that are institutionalized. Previous studies have also concluded that social support settings such as congregate feeding sites provide the greatest nutritional status outcome (9). Congregate feeding sites have been shown to provide the elderly with affordable, healthy food options, resulting in improved nutritional status (9). From these results we can determine that there is an association between independent living while attending a congregate feeding site and good nutritional status.

Further research is needed to discover what specifically supports good nutritional status in independent living individuals and to pinpoint why institutionalized elderly have higher rates of malnutrition. Individuals in nursing homes and assisted living require a higher level of care than individuals living independently and they typically have more medical concerns that can affect nutritional status. Since institutionalized elderly are cared for daily by a medical team and skilled staff, the results of higher rates of malnutrition were unexpected.

There were some weaknesses and limitations in this study. One weakness was the limited relevance of the MNA survey. The 3 different nutritional support settings had very different participant characteristics and therefore not all of the survey questions were relevant. In future research, the use of a different malnutrition survey instrument should be considered. Another potential weakness of this study was its reliance on selfreported information from the participant. Individuals may not tell the truth about their current status when conversing with a medical professional. Using nursing staff and medical chart records could decrease inaccurate self-reporting when collecting data for two of the nutritional settings, but would still prove difficult for individuals living independently with limited medical oversight. Another weakness that could have skewed the results of this study is that the BMI was not used consistently for all participants to determine their final nutritional status score. The independent living group of participants who attended a congregate feeding site had no recorded weight and height on file, resulting in the use of calf circumference in place of BMI. Although this is an acceptable measurement to use according to

Table 2

Nutritional Context: Nutritional Status by Type of Living Facility

\begin{tabular}{|c|c|c|c|c|c|c|}
\hline & \multicolumn{2}{|c|}{ Nursing home } & \multicolumn{2}{|c|}{ Assisted Living } & \multicolumn{2}{|c|}{$\begin{array}{l}\text { Home with Congregate } \\
\text { feeding site }\end{array}$} \\
\hline & Observed & Expected & Observed & Expected & Observed & Expected \\
\hline Normal Nutritional Status & 12 & 14.7 & 12 & 14.7 & 20 & 14.7 \\
\hline At risk or Malnurished Nutritional Status & 13 & 10.3 & 13 & 10.3 & 5 & 10.3 \\
\hline
\end{tabular}

$\mathrm{x} 2(2)=7.04, \mathrm{p}<.05$ 
the MNA, future researchers should standardize the BMI component and directly measure height and weight of all participants for more accurate and consistent results. A limitation of this study was the lack of diversity within the participant group (Table 1). Most of the participants were Caucasian. To ensure that the results are generalizable to the elderly population, future research should incorporate greater diversity in study participants.

\section{Conclusions}

From the results of this study, it was determined that individuals living in a nursing home or assisted living facility could actually be "at risk for malnutrition" or "malnourished" based on the MNA survey. The nursing home residents and assisted living residents showed the highest prevalence of "at risk for malnutrition" or "malnourished" individuals when compared with individuals who live independently, but use congregate feeding. The results of this study help identify the importance of regular nutrition assessments and followups within living institutions to improve the nutritional status among the elderly. This research also sets the framework for future studies to use more variables and compare results at a nominal level.

Future research can go in-depth and look at variables such as caloric intake, number of meals eaten per day, amount of weight lost over a specific period of time, and number of nutrition supplements consumed per day. Variables such as these will help in understanding why institutionalized elderly have the highest rate of malnourishment. This can also help to determine the main contributors of malnutrition in the growing elderly population.

\section{References}

1. Administration on Aging (AoA). (n.d.). Retrieved November 16,2014, from http:// www.Aoa.acl.gov/Aging_Statistics/index.aspx

2. Meijers, J. Malnutrition in Care Home Residents. The Journal of Nutrition, Health \& Aging, 18, 2013.

3. Bagshaw, S. M., \& McDermid, R. C. The role of frailty in outcomes from critical illness. Curr Opin Crit Care, 2013;19(5), 496-503. doi: 10.1097/ MCC.0b013e328364d57

4. Chavarro-Carvajal, D., Reyes-Ortiz, C., Samper-Ternent, R., Arciniegas, A. J., \& Gutierrez, C. C. Nutritional Assessment and Factors Associated to Malnutrition in Older Adults: A Cross-Sectional Study in Bogota, Colombia. J Aging Health, 2014. doi: 10.1177/0898264314549661

5. Kozáková, R., \& Zeleníková, R. Assessing the nutritional status of the elderly living at home. European Geriatric Medicine, 2014;5(6), 377-381. doi: 10.1016/j.eurger.2014.07.003

6. van Bokhorst-de van der Schueren, M. A., Guaitoli, P. R., Jansma, E. P., \& de Vet, H. C. A systematic review of malnutrition screening tools for the nursing home setting. J Am Med Dir Assoc, 2014;15(3), 171-184. doi: 10.1016/j. jamda.2013.10.006

7. Help Guide. A Guide to Nursing Homes, Skilled Nursing Facilities and Convalescent Homes. http: / / www.helpguide.org/articles/senior-housing/ guide-to-nursing-homes.htm. December 2014. Accessed February 2014;17, 2015.

8. The Assisted Living Federation of America. Assisted Living. http://www. alfa.org/alfa/Assisted_Living_Information.asp. 2013. Accessed February 17, 2015.

9. Sylvie, A. K., Jiang, Q., \& Cohen, N. Identification of environmental supports for healthy eating in older adults. J Nutr Gerontol Geriatr, 2013;32(2), 161-174. doi: $10.1080 / 21551197.2013 .779621$ 